\title{
Surface Defect Detection and Classification Based on Statistical Filter and Decision Tree
}

\author{
Habibullah Akbar, Nanna Suryana, and Fikri Akbar
}

\begin{abstract}
Industrial quality inspection is a major issue due to the growing of market competitiveness which requires the product to be checked in terms of online defect detection. Meanwhile, labor inspection has been eliminated due to its limitation that restricts the speed of manufacturing process. Hence, automated inspection process is inevitable to preserve the industrial health and lift human function into management tasks. There are huge efforts on Automated Visual Inspection (AVI) research area, particularly in plain surfaces such as ceramics and fabrics. The inspection modeling includes statistical-based, model-based and color analysis. Most systems are well studied and tested on Charge-Coupled Device (CCD) image sensor. However, only few approaches are carried out for Complementary Metal Oxide Semiconductor (CMOS) imaging modality. This study presents an inspection scheme to detect defect in plain fabric based on statistical filter and geometrical features on CMOS-based image input. The advantage of this technology is obvious regarding to its affordable development especially for small and medium industries. We showed that it is suitable for defect inspection applications that does not require specialized lighting environment. In addition, a classification approach is developed based on decision tree framework. The result for static image shows the classification achieve $99 \%$ accuracy.
\end{abstract}

Index Terms-Automated visual inspection, plain surfaces, statistical filter, thresholding, geometrical moments, and decision tree classifier.

\section{INTRODUCTION}

Human labor has extremely well capabilities to check quality of a product or manufacturing processes under dynamic environment condition where lighting, vibration and temperature can easily change. Traditionally, after the production processes, samples of part are taken for quality assessment. The part that exhibits significant defect will be dissociated while the acceptable part can enter the next process. In fact, human labors suffer with physical limitation when doing the similar job. Consequently, they tend to perform poorly for quality inspection tasks. It has been reported that a man can detect no more than $60 \%$ of the present defects [1].

On the other hand, modern market could easily select

Manuscript received November 15, 2012; revised March 5, 2013. This work was supported in part by the Ministry of Higher Education Malaysia under Fundamental Research Grant Scheme FRGS/2007/FTMK (5)-F0052.

A. Habibullah and Fikri Akbar are with the Faculty of Information and Communication Technology, Universiti Teknikal Malaysia Melaka, Melaka Malaysia (e-mail: habibrown@gmail.com, fikripunya@gmail.com).

Nanna Suryana is with the International Office and lecturer at Faculty of Information Technology and Communication (FTMK) at Universiti Teknikal Malaysia Melaka, Melaka, Malaysia (e-mail: nsuryana@utem.edu.my). another company if they are not happy with the quality of a product. Hence, automated inspection process is inevitable to preserve the industrial health and lift human function into management tasks. Indeed, the Automated Visual Inspection (AVI) to increase product quality has been addressed since four decades ago. There are huge efforts on AVI research area, particularly in plain surfaces such as ceramics and fabrics. The inspection modeling includes statistical-based, model-based and color analysis.

Kumar suggests three categories of automated surface defect detection technique which are statistical, spectral and model-based and noted that spectral approach constitutes the largest number of fabric defect detection in literature [2]. On the other hand, five major categories which are statistical, structural, filter-based, model-based and color texture analysis have been identified in [3]. The author noted that statistical and filter-based approaches have been the most popular method. However, most of their approaches are only well studied and tested on Charge-Coupled Device (CCD) image sensor. Only few approaches are carried out for Complementary Metal Oxide Semiconductor (CMOS) imaging modality.

This study is concern of feasibility of the CMOS-based sensor technology such as webcam to perform surface inspection under uncontrolled lighting environment. Similar to [1], we used statistical-based approach to model the defect-free pattern in the defect detection method. Then any defect can be segmented out based on the modeled pattern. In addition, decision tree classifier is utilized to predict the type of defect.

\section{PREVIOUS WORKS}

The typical AVI structure mainly contains three components which are camera, monitor and part handling controller. In [4], it had been addressed that AVI system covers the full range of technical difficulties in computer vision. The system requires a combination of many aspects of other fields such as computer vision techniques and artificial intelligence. In this study, we address three of them including the acquisition technology, defect detection method and defect classification method.

Many AVI developments have been using CCD-based camera [5]-[7]. This is because it has clear advantage such as low noise and fast shuttering speed. However it has some problems such as high system complexity, high voltage and can only produce analog output signal which requires an additional hardware such as framegrabber to provide digital image for subsequent processing. On the contrary, CMOS camera has low system complexity, low voltage and produces 
digital image and thus can be used directly by computer. However, it produces more noises and poor shuttering speed which causes some blurring effect in the captured images.

This is one of the main reasons why CMOS-based camera has been rarely used for visual inspection in comparison to other imaging modalities. Only few publications indicate the potential of this type of camera for AVI system. As an example, [8] applied CMOS camera into Field Programmable Gate Array (FPGA) chip to inspect the centre position of CD/DVD duplicator. Moreover, [9] had proposed inspection system for satin glass based on Personal Computer (PC) and several CMOS cameras.

On the other hand, statistical-based framework is ubiquitous in image processing literatures [10]-[13]. Until now, there are numerous proposed surface defect detection method based on statistical analysis. The method includes spatial and frequency domain processing. Kumar used linear finite impulse response (FIR) filter with optimized energy separation and explore influence of filter size to detect defect in fabric [14]. Here, ratio between average feature values of filter response is used as quality measure for feature separation. This criterion function is proposed by Singh to optimize linear FIR filter [15]. However, large filter size leads to higher computational cost. On the contrary, bilevel thresholding and morphological filtering are showing good results [1]. A recent survey for surface defect detection methods can be found in [2].

However, modern AVI system has other challenge beyond the defect detection problems which is the defect classification issues that remains an open problem [16]. In contrast to defect detection method, defect classification method is not very common for AVI systems. Most researchers only study the surface defect detection problem without further classifying its type [2], [3]. Recently, AVI trend is moving towards to real-time defect classification. Some intelligent approaches are proposed such as Support Vector Machine (SVM) [17] and neural network [1]. Promising results are described from Neural Network (NN) classifier that combines geometric and statistical features. This study proposed similar approach but using Decision Tree (DT) classifier instead of using NN classifier.

\section{SURFACE DEFECT INSPECTION}

In this study, the approach for fabric defect detection based on statistical filter is presented. The block diagram of surface defect detection is shown in Fig. 1. In this approach, statistical filter is used to segment defective region within defected samples based on two defect-free templates. Each defected class samples is processed to extract its geometrical descriptors. These descriptors are used as input for the DT classifier to predict the class of the defect type. Detail of the inspection stages are described in the following subsequent section.

\section{A. Statistical Filter}

Statistical-based filtering operation is the first process after image is captured as shown in Fig. 2. This process aims to estimate the lower threshold $T_{l}(j)$ and higher threshold $T_{h}(j)$ parameters for each pixel $R(j)$ based on a set of defect-free images. These thresholds are used to detect the existence of the defect.

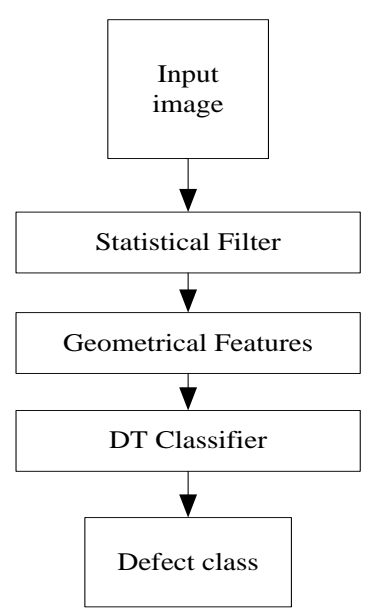

Fig. 1. Block diagram of the proposed surface defect detection method

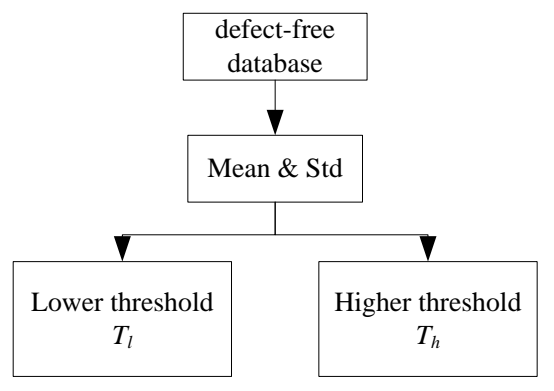

Fig. 2. Statistical filter stage.

This operation is performed based on pixel-by-pixel processing. Assume that there exists $N$ number of defect-free images, then we can calculate the mean and standards deviation measures for each pixel. The higher $T_{h}(j)$ and lower $T_{l}(j)$ thresholds are obtained by using equation (1). The coefficient $\rho$ is the real constant that controls the range between higher and lower threshold. These thresholds are calculated for each pixel $j$ that includes all defect-free images $R_{s}(0<s<N)$.

$$
T_{h, l}(j)=\operatorname{mean}\left(R_{s}(j)\right) \pm \rho \cdot \operatorname{mean}\left(\operatorname{STD}\left(R_{s}(j)\right)\right.
$$

The mean and standard deviation (STD) are calculated by using following formulas.

$$
\begin{gathered}
\text { mean }=\frac{1}{n} \sum_{i=1}^{n} x_{i} \\
\mathrm{STD}=\left(\frac{1}{n} \sum_{i=1}^{n}\left(x_{i}-\bar{x}\right)^{2}\right)^{\frac{1}{2}}
\end{gathered}
$$

The output from this operation is two defect-free template images which correspond to higher and lower thresholds. These images provide the defect-free image model which can filter out the defective area. Therefore, the defective image can be evaluated based on this model. The estimation of defect area pixel $R_{d}$ from defect samples is performed by using the following equation.

$$
R_{d}(j)= \begin{cases}\frac{255,}{0,} & R_{d}(j) \geq T_{h}(j) \text { and } R_{d}(j) \leq T_{l}(j) \\ \text { otherwise }\end{cases}
$$


Defective pixel that has higher value than $T_{h}(j)$ and lower than $T_{l}(j)$ will be set as white pixel (255 is maximum value of 8 -bit intensity image). Otherwise, the nondefective pixel is set as black pixel. We can also see this formula as filtering operation for the test image under two template images. The output from this operation is a binary image $b(i, j)$.

Subsequently, the image is filtered by using erode and average operators to attenuate the noises that are introduced during the acquisition process as shown in Fig. 3.

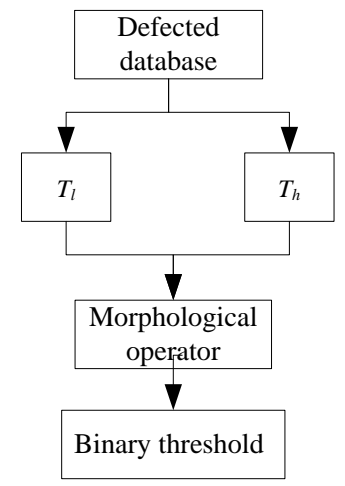

Fig. 3. Noise attenuation stage.

The erode operator is describe in equation (5). The image $b(i, j)_{-S}$ represents the translation of $b(i, j)$ by the point $-s$ where $S E S E$ where parameter $S E$ is the structuring element [18].

$$
b(i, j)_{e}=b(i, j) \otimes S E=\cap_{S \in S E} b(i, j)_{-S}
$$

The binary image $b(i, j)_{e}$ is the output image after erode operation. The next noise filtering process is performed by using average operator. Note that if the correlation kernel is the $m \times n$ matrix $W$, then the average operator is defined as follow.

$$
b(i, j)_{a}=\sum_{n=-k}^{k} \sum_{m=-k}^{k} W(m, n) \cdot b(i+m, j+n)_{e}
$$

The binary image $b(i, j)_{a}$ is the output image after average operation. Finally, the segmentation is performed by using bilevel thresholding $T_{s}$ as follows.

$$
s(i, j)= \begin{cases}\frac{255,}{0,} & \text { if } b(i, j)_{a} \geq T_{s} \\ \text { otherwise }\end{cases}
$$

\section{B. Extraction of Feature}

Although many features are available in literatures, geometrical features are among the strongest descriptors in pattern recognition. The geometrical features such as perimeter $\tau$, area $\sigma$, and circularity $\Phi$ are used for classification purpose due its simplicity and low computational complexity. These features are used as the decision node. They are calculated by the following formulas [19].

$$
\begin{gathered}
\tau=\sum_{i, j=\text { boundary pixel }} f(i, j) \\
\sigma=\sum_{i, j=\text { all-position }} f(i, j) \\
\Phi==\frac{\tau^{2}}{\sigma}
\end{gathered}
$$

The $f(i, j)$ is the intensity value of pixel at row $i$ and column $j$.

\section{Defect Classification}

The decision tree classifier combines the geometric features to get the optimum performance. In this study, there are four classes to be classified i.e. defect-free, dot, vertical and horizontal. The free defect type means there is no defect present in the image. In contrast, the dot, horizontal and vertical defect types means that the defect is present in the image. Since there are four types of classes to be classified, we need to use at least three nodes as represented in Fig. 4.

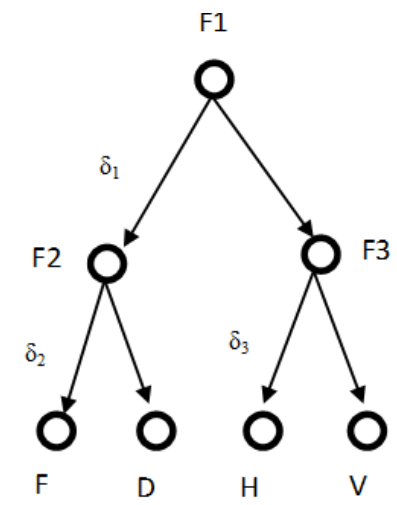

Fig. 4. Decision tree classifier to detect four type of defect classes i.e. F, D, H and V

Parameter $F x$ represents the features as the decision nodes where $x=\{1,2,3\}$. The symbols of $F, D, H$ and $V$ represents defect-free, dot, horizontal and vertical class (defect type) respectively. The if-then rules at each node are used to separate the defect classes as described in the following pseudocode.

$$
\begin{gathered}
\text { if } F 1<\delta_{1} \\
\text { if } F 2<\delta_{2} \\
\text { type }=F \\
\text { else } \\
\text { type }=D \\
\text { end } \\
\text { else } \\
\text { if } F 3<\delta_{3} \\
\text { type }=H \\
\text { else } \\
\text { type }=V \\
\text { end } \\
\text { end }
\end{gathered}
$$

Fig. 5. Pseudocode of the decision tree classifier

\section{EXPERIMENTAL RESULTS}

Four sets of plain fabric were evaluated using the proposed scheme. There are 50 defect-free samples and 191 defective samples including $D, V$, and $H$ defect classes. The typical cropped image samples used in this experiment is shown in Fig. 6 . The image was captured at by using webcam at size of $320 \times 240$ pixels under uncontrolled lighting environment. The computer used for simulation was Pentium D $2.80 \mathrm{GHz}$ 1GB RAM. The algorithm was implemented in Matlab version 7.8 . 


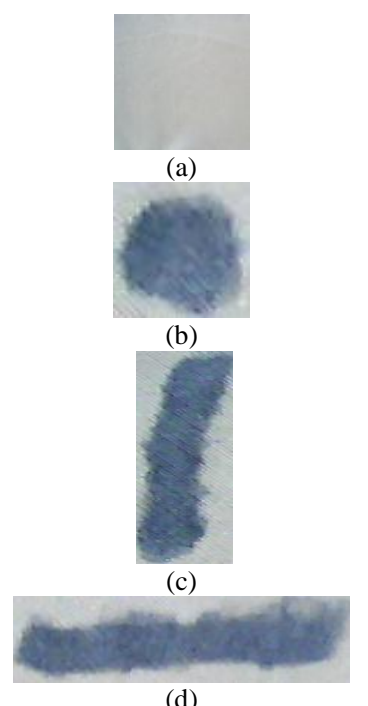

Fig. 6. Sample of our dataset including (a) F, (b) D, (c) V, and (d) H classes.

In defect detection method, the defect-free images dataset was used to obtain two template images based on the higher threshold $T_{h}$ and lower threshold $T_{l}$. These thresholds values were computed based on mean and standard deviation with optimum sensitivity of parameter $\rho$. The value was set as 5 that was judged by experiment with $3 \times 3$ filter size. To remove the acquisition noises, the images were enhanced by using erode and average operators. The structuring element of $1 \times 1$ for erode operator produce better results. Then, the kernel size $W$ of $3 \times 3$ for average operator was confirmed in the experiment. The binary image segmentation was performed using binary thresholding operator. The defect detection results of the three defect classes are shown Fig. 7. The defect area is represented as white area whereas the nondefective area is represented as black pixel. Notice that the defect-free image will contains only black pixel since their intensity values are between the two template images.

The table 1 shows the values of statistical evaluation of three geometrical features that were evaluated in terms of mean $M$, standard deviation STD and Width (length between maximum and minimum values of the distribution as shown in Fig. 8). The $\tau, \sigma$ and $\Phi$ symbols represent perimeter, area and circularity features respectively. The mean of perimeter feature for $D$ class is smaller than mean for $H$ and $V$. The lowest $S T D$ was given by $D$ which corresponds to its Width.

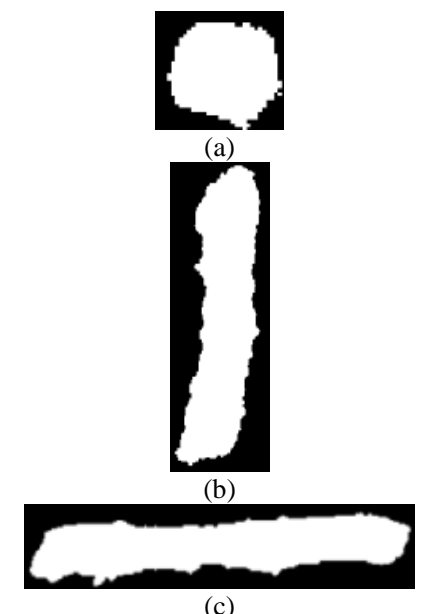

(c)

Fig. 7. Results of the defect detection scheme: (a) $D$, (b) $V$, and (c) $H$.
On the other hand, the mean of area feature has significant different values for each class. This feature is useful to distinguish the three classes of defect type. In contrast to area feature, the mean of circularity feature not only provide different values for each class, but also maintain very low $S T D$ values.

TABLE I: ThreE Extracted FeAtures by MEANS OF MEAN M, STANDARD DEVIATION STD AND WIDTH W

\begin{tabular}{|c|c|c|c|c|}
\hline Feature & Type & $M$ & $S T D$ & Width \\
\hline \multirow{3}{*}{$\tau$} & $D$ & $1.08 \mathrm{E}+02$ & $1.37 \mathrm{E}+01$ & $4.24 \mathrm{E}+01$ \\
\hline & $H$ & $5.17 \mathrm{E}+02$ & $2.79 \mathrm{E}+01$ & $1.15 \mathrm{E}+02$ \\
\hline & $V$ & $5.65 \mathrm{E}+02$ & $4.64 \mathrm{E}+01$ & $1.54 \mathrm{E}+02$ \\
\hline \multirow{3}{*}{$\sigma$} & $D$ & $8.33 \mathrm{E}+02$ & $1.88 \mathrm{E}+02$ & $5.67 \mathrm{E}+02$ \\
\hline & $H$ & $5.88 \mathrm{E}+03$ & $6.87 \mathrm{E}+02$ & $2.65 \mathrm{E}+03$ \\
\hline & $V$ & $9.01 \mathrm{E}+03$ & $1.28 \mathrm{E}+03$ & $4.11 \mathrm{E}+03$ \\
\hline \multirow{3}{*}{$\Phi$} & $D$ & 8.88E-01 & $3.39 \mathrm{E}-02$ & $1.24 \mathrm{E}-01$ \\
\hline & $H$ & $2.77 \mathrm{E}-01$ & $2.51 \mathrm{E}-02$ & $1.08 \mathrm{E}-01$ \\
\hline & $V$ & $3.53 \mathrm{E}-01$ & $1.12 \mathrm{E}-02$ & 4.59E-02 \\
\hline
\end{tabular}

Fig. 8 represents the visual distribution of the samples used in this experiment whereas the vertical-axis describes four defect classes: 0 as $F, 1$ as $D, 2$ as $H$ and 3 as $V$.

As shown in Fig. 8, perimeter feature is useful to distinguish between defects classes of $D$ and $H$ due to their high inter-class variability. In contrast, the $H$ and $V$ intra-class variability is large whereas most of their values are overlapping. This feature cannot be used to distinguish the $H$ and $V$ defect classes. In contrast to perimeter, area features values are useful to distinguish between for all defect classes because their high inter-class variability. Although some values from $H$ and $V$ are overlapping, most of their values are well separated. Similar to perimeter feature, it has small intra-class variability for $D$ defect class. On the other hand, the circularity features values provide moderate inter-class variability for all classes. This feature also provides small intra-class variability for each defect type. Therefore, this feature is useful to distinguish the three defect classes. Notice that the class $F$ has no value for any features because the defective pixel is not present.

Then, these features were used to develop the DT classifier. Since perimeter feature cannot be used to distinguish between $\mathrm{H}$ and $\mathrm{V}$ defect classes, then this feature should be used as $F 1$. The threshold $\delta_{\tau}$ value was obtained from the mean between D and others ( $\mathrm{H}$ and $\mathrm{V}$ ) which is 325 . This feature separates the $F$ and $D$ defect class from $H$ and $V$ defect classes. Indeed, the area feature is similar to perimeter which also can be used as the first-level node $F 1$ as shown in Fig. 4. However, it has to be used for the rest features i.e. F2 or F3. Since this feature has small intra-class and moderate inter-class variabilities for $F$ and $D$, then it is suitable for the second-level node $F 2$ to separate them. The threshold $\delta_{\sigma}$ value was obtained from the mean between $F$ and $D$ is 416 . The circularity feature $\Phi$ can 
be used to distinguish between all defect types. Therefore, this feature is suitable for the second-level node $F 3$. The threshold $\delta_{\Phi}$ was obtained from the mean between $H$ and $\mathrm{V}$ is 0.315 . To evaluate the decision tree classifier, the output of the decision tree classifier was compared with the labeled features. The classifier was able to detect samples in accuracy of $99 \%$.

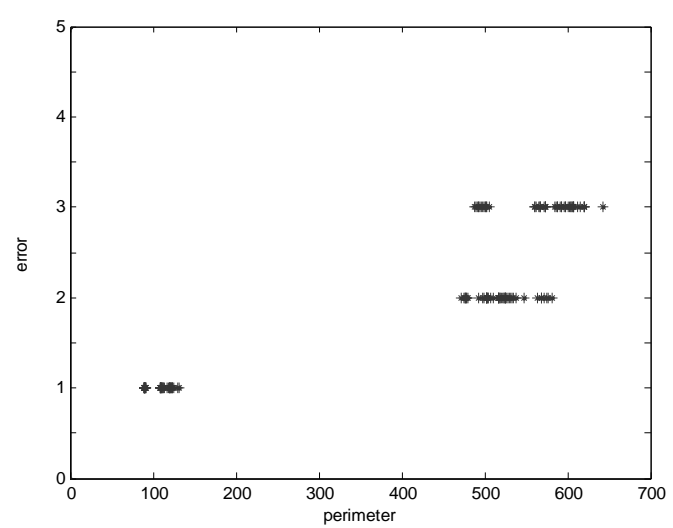

(a)

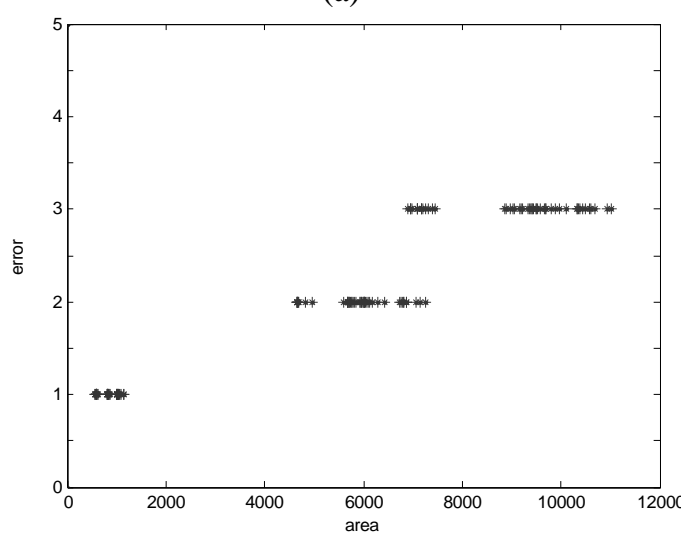

(b)

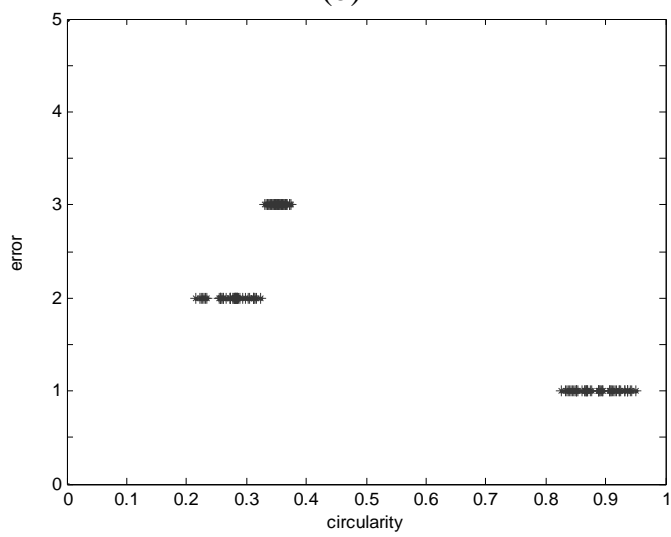

(c)

Fig. 8. Feature data distributions of (a) Perimeter, (b) Area, and (c) Circularity

\section{CONCLUSION}

In this research, an inspection scheme is proposed to segment and classify defect type in plain fabric. The defect detection is based on statistical filter, erode, average and bilevel thresholding while the classification is based on geometrical features and decision tree classifier. The mean and standard deviation measures are calculated to obtain higher and lower thresholds parameters to provide a reference of defect-free model. The thresholds were used to filter the defective area from nondefective area. The noises in the image were attenuated using erode and average filter. Then, the defective area is segmented by using bilevel thresholding. The result shows that all defective samples are detected.

After segmentation, three geometrical features were extracted to characterize the defect types. These features were statistically evaluated based on the mean, standard deviation and width measures and used to develop the decision tree classifier. The result showed that the decision tree classifier was able to detect $99 \%$ of the samples. The image used in this research is captured in static condition under uncontrolled lighting environment. However, the real industrial environment requires the image to be captured while moving in the production lines. Further studies to perform plain fabric inspection under real production condition are suggested.

\section{ACKNOWLEDGMENT}

The authors thank to Dr. Anton Satria Prabuwono for the support and useful ideas of this study.

\section{REFERENCES}

[1] R. Stojanovicw, P. Mitropulos, C. Koulamas, Y. Karayiannis, S. Koubias, and G. Papadopoulos, "Real-time vision-based system for textile fabric inspection," Real-Time Imaging, vol. 7, no. 6, pp. 507-518, 2001.

[2] A. Kumar, "Computer-vision-based fabric defect detection: a survey," IEEE Trans. on Industrial Electronics, vol. 55, no. 1, pp. 348-363, 2008.

[3] X. Xie, "A review of recent advances in surface defect detection using texture analysis techniques," Electronic Letters on Computer Vision and Image Analysis, vol. 7, no. 3, pp. 1-22, 2008

[4] J. M. Bowskill, T. Katz, and J. H. Downie, "Solder inspection using an object-oriented approach to machine vision," in Proc. SPIE of Machine Vision Application in Industrial Inspection III, 1995, pp. 34-35.

[5] C. Fernandez, J. Suardiaz, C. Jimenez, P. J. Navarro, A. Toledo, and A. Iborra, "Automated visual inspection system for the classification of preserved vegetables," in Proc. of the International Symposium on Industrial Electronics, pp. 265-269, 2002.

[6] C. Fernandez, C. Platero, P. Campoy, and R. Aracil, "Vision system for on-line surface inspection in aluminum casting process," in Proc. of the International Conference on Industrial Electronics, Control, and Instrumentation, pp. 1854-1859, 1993.

[7] W. Yang, D. Li, X. Wei, Y. Kang, and F. Li, "An automated visual inspection system for foreign fiber detection in lint," in Proc. of the Global Congress on Intelligent Systems, 2009, pp. 364-368.

[8] Y. C. Chen and Y. T. Wang, "Development of a low-cost machine vision system and its application on inspection processes," Tamkang Journal of Science and Engineering, vol. 11, no. 4, pp. 425-431, 2008.

[9] F. Adamo, F. Attivissimo, A. Di Nisio, and M. Savino, "A low-cost inspection system for online defects assessment in satin glass," Measurement, vol. 42, no. 9, pp. 1304-1311, 2009.

[10] T. Ojala, M. Pietikainen, and T. Maenpaa, "Multiresolution gray-scale and rotation invariant texture classification with local binary patterns," IEEE Trans. on Pattern Analysis and Machine Intelligence, vol. 24, no. 7, pp. 971-987, 2002.

[11] J. M. Francos, A. Z. Meiri, and B. Porat, "A unified texture model based on a 2-D Wold-like decomposition," IEEE Trans. on Signal Processing, vol. 41, no. 8, pp. 2665-2678, 1993.

[12] C. Neubauer, "Segmentation of defects in textile fabric," in Proc. $11^{\text {th }}$ International Conference on Pattern Recognition: Computer Vision and Applications, 1992, pp. 688-691.

[13] D. M. Tsai and C. Y. Hsieh, "Automated surface inspection for directional textures," Image and Vision Computing, vol. 18, no. 1, pp. 49-62, 1999.

[14] A. Kumar and G. K. H. Pang, "Defect detection in textured materials using optimized filters," IEEE Trans. on Systems, Man, and Cybernetics, Part B, vol. 32, no. 5, pp. 553-570, 2002.

[15] H. Singh and A. Mahalanobis, "Correlation filters for texture recognition and applications to terrain-delimitation in wide-area surveillance," in Proc. International Conference on Acoustics, Speech and Signal Processing, 1994, pp. 153-156. 
[16] M. Shirvaikar, "Trends in automated visual inspection," Journal of Real-Time Image Processing, vol. 1, no. 1, pp. 41-43, 2006.

[17] X. G. Zhang, J. J. Xu, and G. Y. Ge, "Defects recognition on X-ray images for weld inspection using SVM," in Proc International Conference on Machine Learning and Cybernetics, 2004, pp. 3721-3725.

[18] R. M. Haralick, S. R. Sternberg, and X. Zhuang, "Image analysis using mathematical morphology," Transactions on Pattern Analysis and Machine Intelligence, no. 4, pp. 532-550, 1987.

[19] O. Soderkvist, Computer Vision Classification of Leaves from Swedish Trees, Master Thesis, Linkoping University, 2001.

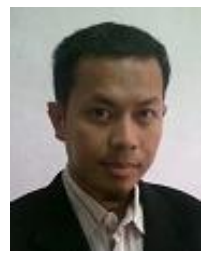

Habibullah Akbar received the bachelor of science in Physics at Institut Teknologi Bandung (Bandung, Indonesia) in 2006. He received Master of Science in Information and Communication Technology (ICT) a Universiti Teknikal Malaysia Melaka (UTeM) in 2010. His research interests include computer vision, robotics, and electronics.

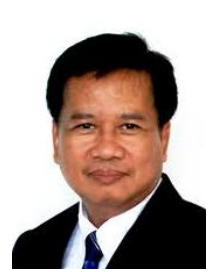

Nanna Suryana has received his bachelor of science in soil and water eng. (Bandung, Indonesia), M.Sc. in Comp. Assisted for Geoinformatics and Earth Science, (Enschede, Holland), Ph.D. in Geographic Information System (GIS) (Wageningen, Holland). He is currently holding a position of Director of International Office and lecturer at Faculty of Information Technology and Communication (FTMK) of Universiti Teknikal Malaysia Melaka (UTEM). His current research interest is in field of GIS and Data Mining.

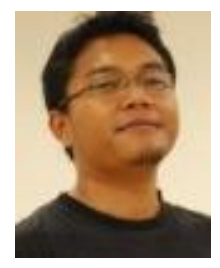

Fikri Akbar received the bachelor of information technology, security technology at Multimedia University (Melaka, Malaysia) in 2008. He is currently pursuing Master of Science in Information and Communication Technology (ICT) at Universiti Teknikal Malaysia Melaka (UTeM). His research interests include remote sensing image compression and classification. 